\title{
Compact Binaries as Tracers of Star Formation Histories: the XMM Survey of M31
}

\author{
U. Kolb, J. Osborne and M.G. Watson \\ Dept. of Physics \& Astronomy, University of Leicester, \\ Leicester LE1 7RH, UK
}

\begin{abstract}
X-ray binaries (XBs) dominate the X-ray emission of normal galaxies. The new X-ray satellite XMM will study the XB population of M31 in detail. The resulting M31 sample will significantly advance our understanding of the evolutionary history of XBs, and ultimately allow us to probe the star formation history of stellar populations by X-ray observations.
\end{abstract}

\section{Introduction}

The most efficient energy source in the Universe is accretion onto compact objects, i.e. the release of gravitational energy when mass falls down the potential well of a black hole or neutron star. Galactic compact binaries (XBs), where a normal star supplies a steady stream of material to a compact companion, appear therefore as the brightest sources in the X-ray sky, with X-ray luminosities up to $10^{39} \mathrm{erg} \mathrm{s}^{-1}$ (for a recent review see, e.g. van Paradijs 1998). These highenergy laboratories offer not only an insight into the exciting physics of radiation and matter under extreme conditions, the present $\mathrm{XB}$ population also carries information on the various phases in their evolutionary history, in particular an imprint of the star formation history in the considered stellar ensemble.

The Galactic XB sample $(\simeq 200$ systems; $\simeq 50$ with determined orbital period; see, e.g. van Paradijs 1995, Ritter \& Kolb 1998) is not well suited to extract this information. The sample is inhomogeneous and incomplete; the distances to individual systems and their luminosities are uncertain.

Modern imaging X-ray instruments are now sensitive enough to resolve the $\mathrm{X}$-ray active population of nearby galaxies. With XBs as the brightest sources a more detailed study of the XB population in galaxies other than our own is feasible. In the following we report on a comprehensive X-ray survey of M31, to be conducted with the European X-ray satellite XMM which is due for launch in early 2000, and on how this will advance our understanding of XB physics.

\section{The X-ray Content of Normal Galaxies}

The integrated X-ray output of normal galaxies typically is $39 \lesssim$ $\log L_{X} / \mathrm{erg} \mathrm{s}^{-1} \lesssim 41$ for spirals and $39 \lesssim \log L_{X} / \mathrm{erg} \mathrm{s}^{-1} \lesssim 43$ for ellipticals. Emission from a hot interstellar medium dominates the X-ray brightest ellipticals. A simple extrapolation of our own Galaxy's X-ray content suggests that 
XBs contribute about two thirds to the X-ray flux of spirals, while one third is from normal stars and diffuse emission (e.g. Watson 1990, Fabbiano 1995, Fabbiano 1996).

The main classes of discrete X-ray sources and corresponding typical X-ray luminosities (in $\left.\log L_{X} / \mathrm{erg} \mathrm{s}^{-1}\right)$ are: soft X-ray transients $(37-39$ in outburst), persistent low-mass X-ray binaries $(35-38)$, high-mass X-ray binaries $(35.5-38$ in outburst, $32-34$ in quiescence), supernova remnants $(34.5-35.5)$, cataclysmic variables $(29-34)$, RS CVn stars $(30-31)$, normal stars $(27-28$; although $\mathrm{X}$-ray active stars may be as bright as $32-33)$ and supersoft binaries (36-38, but in the soft X-ray band).

M31 will prove ideal for testing such estimates.

\section{Previous X-ray Studies of M31}

Knowledge of M31 in X-rays has improved markedly with the completion of two ROSAT surveys of this galaxy (see, e.g. Fabbiano 1995 for a summary of the pre-ROSAT account). ROSAT observed six fields with $26-49$ ks exposure time in 1991, covering roughly 2.5 times the area of M31's $D_{25}$ ellipse (Supper et al. 1997), and 80 overlapping fields with $2.5 \mathrm{ks}$ each in 1992, the centres of which form a dense grid covering the $D_{25}$ ellipse (Supper 1995). The corresponding detection limit for a M31 point source was $5 \times 10^{35} \mathrm{erg} \mathrm{s}^{-1}$ in the ROSAT band $(0.1 \lesssim E / \mathrm{keV} \lesssim 2)$.

Overall, ROSAT detected 560 point sources. Of these, 80 turned out to be foreground objects, 100 background objects. Thirty of the remaining 380 sources intrinsic to M31 coincide with M31 globular clusters, 17 with supernova remnants; 15 sources are "supersoft", i.e. potentially semi-detached white dwarf binaries.

Studies of the diffuse emission face the usual difficulty of removing the contribution of these point sources. Primini et al. (1993) considered the inner bulge (within $\lesssim 1 \mathrm{kpc}$ from the centre) and found a $70-80 \%$ excess emission over the extrapolated $\log N-\log S$ relation. West et al. (1997) specifically excluded this core region because of the severe source confusion there. Further out they found two-component diffuse emission with a spherical core and a disc component with $5 \mathrm{kpc}$ radial scale length. The emission from the disc is consistent with the flux expected from the underlying normal stellar population, assuming a typical optical to X-ray flux ratio $10^{-4}$.

The ROSAT catalog contains 15 "transients", i.e. sources that have been seen in only one ROSAT survey, or have been seen earlier by EINSTEIN and not by ROSAT, or vice versa. About 50 sources are labelled as "variable". Yet to date, the majority of the ROSAT sources is of unknown nature.

Once the new X-ray satellites XMM and AXAF are operational - at the time of writing launch dates are early 1999 (AXAF) and early 2000 (XMM) this situation is certain to improve significantly.

Both XMM and AXAF have imaging capabilities up to $10 \mathrm{keV}$ energy, not just to $2 \mathrm{keV}$ as ROSAT. Crudely, the XMM count rate is 5-20 times higher than for ROSAT, with comparable angular resolution (XMM: 15", ROSAT PSPC 25", ROSAT HRI 5"; all values HEW). AXAF is less sensitive than XMM $(25-50 \%)$ but has a better resolution $\left(0.5^{\prime \prime}\right)$. For a detailed account of technical specifica- 
M31 XMM GT pointings \& Rosat sources

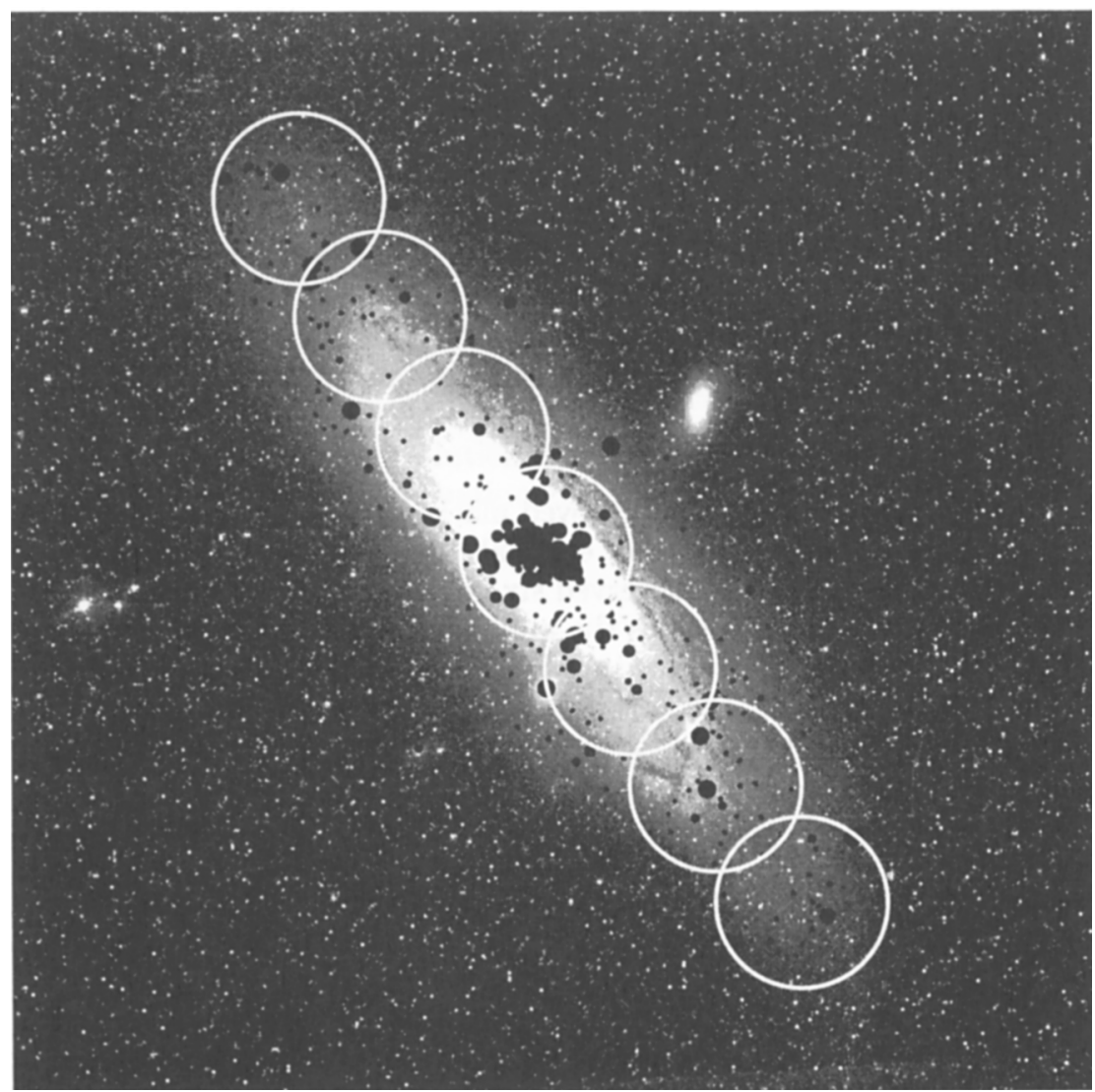

Figure 1. XMM survey fields (white circles) and the ROSAT X-ray sources (black dots; size indicates X-ray intensity) superimposed on an optical image of M31.

tions of instruments aboard XMM and AXAF we refer to, e.g. Lumb et al. 1996 (XMM; see also http://astro.estec.esa.nl/XMM/news/A0/ao1_top.html) and Weisskopf et al. 1996 (AXAF; see also http: //asc.harvard.edu/udocs/docs/docs.html).

\section{The XMM M31 Survey}

A major new survey of M31 is planned with XMM as part of the Guaranteed Time programme of two of the XMM project teams: the XMM Survey Science Centre (SSC) and the Optical Monitor (OM) consortium. The survey will cover seven adjacent fields with $\simeq 30^{\prime}$ diameter, extending in both directions along 
the major axis of M31 (see Fig. 1). ${ }^{1}$ The field centred on the core of M31 will be observed twice to enable variability in the nuclear region to be monitored. The planned exposure time per field is $67 \mathrm{ks}$, pushing the detection limit down to $L_{X} \simeq 3 \times 10^{34} \mathrm{erg} \mathrm{s}^{-1}$ in the $0.5-2 \mathrm{keV}$ band. The XMM survey covers about $65 \%$ of the area of the ROSAT survey, but extrapolating the luminosity function of West et al. (1997) we expect to detect about 4000 sources intrinsic to M31. Significantly, the count rate of the 250 brightest sources $\left(z 10^{36} \mathrm{erg} \mathrm{s}^{-1}\right)$ - the bulk of the XB population - will be high enough for a detailed spectral and time variability study. This will allow us to distinguish between black hole and neutron star accretors, and to determine the orbital period, $P$, of those XBs with orbital modulation of the X-ray flux. We expect to find the period for about 30 systems.

A somewhat less extensive survey of M31 is planned with AXAF (PI: S. Murray, CfA). AXAF observations are of particular importance in the core region where source confusion might be a problem for XMM.

A comparison of ROSAT, XMM and AXAF data allows one to find X-ray transients and constrain the transient rate.

Optical counterparts will be difficult to detect. Assuming a distance modulus of 24.4 mag (e.g. Holland 1998, and references therein) the typical V magnitude of a high-mass or low-mass XB (see below) is $+20 \mathrm{mag}$ and $+29 \mathrm{mag}$, respectively.

\section{X-ray Binary Physics}

The main objective of the M31 XB survey is to provide a statistically clean sample of equidistant systems. Ideally, we would like to constrain the luminosity and period distribution of the various subclasses (neutron star or black hole systems, transient or persistently bright systems) in the different subpopulations of M31 (bulge, disc and spiral arms, as indicated by the projected source location).

Such a sample will provide vital clues in two main areas: the evolutionary histories of XBs themselves - this involves the long-standing mystery of the formation of stellar mass black holes - and ultimately the star formation history of the stellar population these XBs belong to.

XBs appear in two main flavours: systems powered by Roche-lobe overflow, and systems where the compact object accretes from the companion's stellar wind. Low-mass XBs (LMXBs), i.e. systems with a low-mass companion, always belong to the first group, while high-mass $\times$ XBs (HMXBs) with a high-mass companion usually are wind-fed.

XBs powered by Roche-lobe overflow are particularly interesting for evolutionary considerations. Because of Roche geometry and Kepler's law the orbital period is a direct measure of the companion's mean density $(P \propto 1 / \sqrt{\rho})$, hence provides information on its mass and/or evolutionary state. This in turn sheds light on the system's prehistory.

\footnotetext{
${ }^{1}$ The fields south and north of the centre will be observed by the SSC team (PI: J. Osborne) and the OM team (PI: W. Priedhorsky, LANL), respectively.
} 
Prior to the X-ray active phase these XBs must have evolved through two rather violent events. A compact star forms through core collapse of a massive, extended star. Only an initially wide binary - much wider than the compact $\mathrm{XB}$ - can accommodate such a giant star. The required dramatic reduction of the orbital separation occured most likely via a common envelope phase, where orbital binding energy is used to drive off the extended star's envelope. Substantial mass ejection also takes place as a result of the supernova (SN) explosion. In the case of a spherically symmetric SN the binary disrupts if more than half of the total binary mass is lost. Hence XB progenitors with low-mass primaries and relatively massive secondaries have the best survival probability. In particular, the spiral-in and envelope ejection phase (reducing the primary mass) must have occured before the SN. The collapsing star therefore was most likely a helium star (see, e.g. Kalogera \& Webbink 1996, 1998).

Though close, the post-SN binaries are still detached. Two mechanisms compete to establish and maintain the semi-detached X-ray state: nuclear expansion of the secondary ("n-driven" systems) and orbital angular momentum losses $\dot{J}$ (by gravitational wave emission and magnetic stellar wind braking; " $\mathrm{j}$ driven" systems). Stellar evolution theory allows one to calculate the expansion timescale $t_{\text {exp }}$ of n-driven evolution with confidence, whereas the orbital braking timescale $t_{J}$ for $\mathrm{j}$-driven evolution is still uncertain. The expansion time $t_{\exp }$ is short and therefore dominant if the donor is either a fairly massive main-sequence star, or has already left the main-sequence stage. In both cases the donor's mean density is small, thus $P$ long. Hence long-period LMXBs are generally n-driven and evolve to longer periods, while short-period systems are $\mathrm{j}$-driven and evolve to shorter periods (see, e.g. Kolb 1998, and references therein).

An important diagnostic quantity for constraining $t_{J}$ is therefore the ratio of short-period to long-period systems. Crucial in this context are the M31 cousins of the X-ray bright "Galactic bulge sources". So far these Galactic sources escaped classification as their optical counterparts are heavily absorbed, and none of the X-ray lightcurves revealed orbital modulations. They are numerous enough to almost reverse the characteristics of the observed period distribution of Galactic XBs. With essentially all M31 bulge sources in one field and a sufficiently long exposure time to detect periods $\lesssim 1 / 2$ day, XMM and AXAF are likely to finally unveil the nature of these systems.

XBs also differ in the nature of the accretor which can be either a black hole (typically with mass $10 M_{\odot}$ ) or a neutron star (typically with mass $1.4 M_{\odot}$ ).

The neutron star LMXB case is complex but qualitatively understood. Progenitors with the highest chances to survive the SN have rather low-mass helium star primaries which expand to giant dimensions. This requires a relatively wide pre- and post-SN orbit, making the time to evolve to the semi-detached X-ray stage long. By then the donor star has either already terminated core hydrogen burning (and the system is n-driven), or will be close to the end of it. The latter group of systems has been identified with neutron star transients (King \& Kolb 1997). This sensitivity to evolutionary parameters allows one to constrain the mean kick velocity imparted to the neutron star at birth, the strength of orbital braking and the common envelope efficiency from observed global properties like the transient fraction among short-period systems and the ratio of long-period 
to short-period systems (Kalogera, Kolb \& King 1998). Improved statistics will make these constraints tighter.

Much less is known about the formation of black hole binaries (see, e.g. Kalogera 1998 who considered the natural extrapolation of the neutron star $\mathrm{XB}$ channel). The study of black hole XBs is probably the only way to gain understanding of the formation of stellar-mass black holes.

An exciting far-reaching application of the M31 project is its relevance for the star formation history (SFH) of galaxies. As the X-ray active lifetime of HMXBs is of order the massive donor's nuclear time, i.e. $\lesssim 10^{6}-10^{7} \mathrm{yr}$, HMXBs act as indicators for the current star formation rate. In contrast, there is a characteristic time delay of order $t_{J}$, typically several Gyr, between the formation of the progenitor binary and the X-ray active time of a LMXB. Hence LMXBs probe regions of past star formation. White \& Ghosh (1998) pointed out that a possible peak in the cosmic star formation rate (as suggested by Madau et al. 1996, 1998) would therefore lead to a double-peaked X-ray activity as a function of redshift. The M31 survey provides an ideal opportunity to advance our understanding of the intrinsic evolutionary timescale of LMXBs. With this knowledge an independent test of the star formation history in nearby galaxies through X-ray observations is at hand.

Acknowledgements. UK acknowledges an IAU travel grant. Theoretical astrophysics research at Leicester is supported by a PPARC Rolling Grant.

\section{References}

Fabbiano, G. 1996, MPE Report, 263, 347

Fabbiano, G. 1995, in: X-ray Binaries, (eds.) W.H.G. Lewin, J. van Paradijs \& E.P.J. van den Heuvel, Cambridge: CUP, p. 390

Holland, S. 1998, AJ, 115, 1916

Kalogera, V. 1998, ApJ, in press

Kalogera, V., Webbink, R.F. 1996, ApJ, 458, 301

Kalogera, V., Webbink, R.F. 1998, ApJ, 493, 351

Kalogera, V., Kolb, U., King, A.R. 1998, ApJ, 504, 967

King, A.R., Kolb, U. 1997, ApJ, 481, 918

Kolb, U. 1998, MNRAS, 297, 419

Madau, P., Ferguson, H.C., Dickinson, M.E., Giavalisco, M., Steidel, C.C., Fruchter, A. 1996, MNRAS, 283, 1388

Madau, P., Pozzetti, L., Dickinson, M. 1998, ApJ, 498, 106

Lumb, D., Eggle, H., Laine, R., Peacock, A. 1996, Proc. SPIE, 2808, 326

Primini, F.A., Forman, W., Jones, C. 1993, ApJ, 410, 615

Ritter, H., Kolb, U. 1998, A\&AS, 129, 83

Supper, R. 1995, PhD thesis, Ludwig-Maximilians-Universität München

Supper, R., Hasinger, G., Pietsch, W., Trümper, J., Jain, A., Magnier, E.A., Lewin, W.H.G., van Paradijs, J. 1997, A\&A, 317, 328 
Van Paradijs, J. 1998, in: The Many Faces of Neutron Stars, (eds.) R. Buccheri, J. van Paradijs \& M.A. Alpar, Dordrecht: Kluwer, in press, astro$\mathrm{ph} / 9802177$

Van Paradijs, J. 1995, in: X-ray Binaries, (eds.) W.H.G. Lewin, J. van Paradijs \& E.P.J. van den Heuvel, Cambridge: CUP, p. 536

Watson, M.G. 1990, in: Windows on Galaxies, (eds.) G. Fabbiano, J.S. Gallagher \& A. Renzini, Dordrecht: Kluwer, p. 177

Weisskopf, M.C., O'Dell, S.L., Van Speybroeck, L.P. 1996, Proc. SPIE, 2805, 2 West, R.G., Barber, C.R., Folgeraither, E.L. 1997, MNRAS, 287, 10

White, N.E., Ghosh, P. 1998, ApJ, 504, L31

\section{Discussion}

Zijlstra: The resolution of 15 arcsec may make it difficult to identify the counterparts of the X-ray sources.

Kolb: Comparison with AXAF helps as this satellite has 0.5 arcsec resolution. With optical counterparts being so faint we hope to classify the 250 or so brightest X-ray sources by X-ray observations alone (lightcurves, spectra). 